\title{
Simulation-based optimization of coupled material-energy flow at ironmaking-steelmaking interface using One-Ladle Technique
}

\author{
Hu, Z.C. ${ }^{a, b}$, Zheng, Z. ${ }^{\text {a }}{ }^{*}$, He, L.M. ${ }^{c}$, Fan, J.P. ${ }^{b}$, Li, F. $^{\text {b }}$ \\ aCollege of Materials Science and Engineering, Chongqing University, Chongqing, P.R. China \\ ${ }^{b} \mathrm{CISDI}$ Engineering Co., Ltd., Chongqing, P.R. China \\ 'Chongqing College of Electronic Engineering, Chongqing, P.R. China
}

\begin{abstract}
A B S T R A C T
The ironmaking-steelmaking interface of the steel manufacturing process involves the hot metal ladle circulation and the energy dissipation which are coupled processes with an interrelated but independent relation. Therefore, the synergistic operation of the material flow and the energy flow at the interface is momentous to the effective production of the ironmaking-steelmaking section. However, there is a lack of solutions to realize the synergy. Here, we presented a coupling simulation model for the material flow and energy flow of the ironmaking-steelmaking interface, based on the mathematical description of their operation behaviors, the operation and technical model of the production equipment and the temperature-decreasing model of the ladle. Further, the coupling simulation model was applied to a concrete ironmakingsteelmaking interface using the One-Ladle Technique. The coupling simulation model proved its performance in providing comprehensive decisionmaking supports and optimized production management strategies by achieving a solution that results in a decline of $10^{\circ} \mathrm{C}$ in the average temperature drop of the hot metal and a reduction in the cost per tonne of steel by CNY 1.02.
\end{abstract}

\author{
ARTICLE INFO \\ Keywords: \\ Metallurgy; \\ Ironmaking process; \\ Steelmaking process; \\ Ironmaking-steelmaking interface; \\ Coupled material-energy flow; \\ Discrete event simulation; \\ Optimization; \\ One-ladle technique \\ *Corresponding author: \\ zhengzh@cqu.edu.cn \\ (Zheng, Z.)
}

Article history:

Received 8 August 2021

Revised 17 September 2021

Accepted 19 September 2021

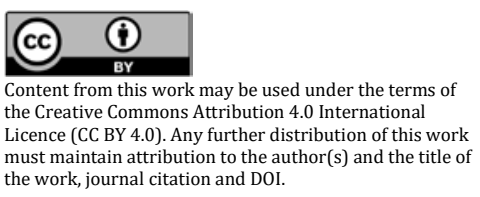

\section{References}

[1] Zhao, J.Y., Wang, Y.J., Xi, X., Wu, G.D. (2017). Simulation of steel production logistics system based on multi-agents, International Journal of Simulation Modeling, Vol. 16, No. 1, 167-175, doi: 10.2507/IJSIMM16(1)C04.

[2] Wang, X., Wei, Z., Wang, S., Zhou, W., Liu, S. (2012). Multi-agent based simulation system modelling for steelmaking-continuous casting production process, In: Proceedings of $20122^{\text {th }}$ Chinese Control and Decision Conference (CCDC), Taiyuan, China, 170-173, doi: 10.1109/CCDC.2012.6242928.

[3] Fan, X.-K. (2010). Hot metal transportation method and general layout of iron \& steel plant, Architectural and Structural Design, No. 10, 29-34.

[4] Li, M.-Y., Yan, Y.-G., Lu, S.-W., Luo, Z.-G. (2016). Process design and simulation of iron-steelmaking zero interface, Shandong Metallurgy, Vol. 38, No. 4, 26-29.

[5] Shang, G., Xiang C., Duan, X. (2017). Research and application of general layout parttern of "one-ladle mode" on ironmaking/steelmaking interface, China Metallurgy, Vol. 27, No. 8, 45-49. 
[6] Wiyaratn, W., Watanapa, A. (2010). Improvement plant layout using systematic layout planning (SLP) for increased productivity, International Journal of Mechanical, Industrial and Aerospace Sciences, Vol. 4, No. 12, 1382-1386, doi: 10.5281/zenodo.1330985.

[7] Penteado, F.D., Ciric, A.R. (1996). An MINLP approach for safe process plant layout, Industrial \& Engineering Chemistry Research, Vol. 35, No. 4, 1354-1361, doi: 10.1021/ie9502547.

[8] Balakrishnan, J., Cheng, C.-H., Wong, K.-F. (2003). FACOPT: A user friendly facility layout optimization system, Computers \& Operations Research, Vol. 30, No. 11, 1625-1641, doi: 10.1016/S0305-0548(02)00096-5.

[9] Qiu, J., Tian, N.-Y. (2005). Relative superiority research on the ironmaking/steelmaking interface of the typical process section, Journal of University of Science and Technology Beijing, Vol. 27, No. 6, 740-744.

[10] Zhang, L.-Q., Tian, N.-Y., Xu, A.-J. (2008). Selection of hot metal pretreatment mode for new steel-making plant, Steelmaking, Vol. 24, No. 1, 58-62.

[11] Tang, L., Wang, G., Liu, J. (2007). A branch-and-price algorithm to solve the molten iron allocation problem in iron and steel industry, Computers \& Operations Research, Vol. 34, No. 10, 3001-3015, doi: 10.1016/j.cor.2005.11.010.

[12] Wang, G., Tang, L. (2007). A column generation for locomotive scheduling problem in molten iron transportation, In: Proceedings of 2007 IEEE International Conference on Automation and Logistics, Jinan, China, 2227-2233. doi: 10.1109/ICAL.2007.4338946.

[13] Yang, F., Shi, M. (2019). Emergency surgery scheduling under urban emergencies based on improved moth-flame optimization, Journal Européen des Systèmes Automatisés, Vol. 52, No. 1, 49-55, doi: 10.18280/jesa.520107.

[14] Su, J., Wei, M., Liu, A. (2018). A robust predictive-reactive allocating approach, considering random design change in complex product design processes, International Journal of Computational Intelligence Systems, Vol. 11, No. 1, 1210-1228, doi: 10.2991/ijcis.11.1.91.

[15] Bedhief, A.O. (2021). Comparing mixed-integer programming and constraint programming models for the hybrid flow shop scheduling problem with dedicated machines, Journal Européen des Systèmes Automatisés, Vol. 54, No. 4, 591-597, doi: 10.18280/jesa.540408.

[16] Su, J., Yang, Y., Yu, K., Zhang, N. (2018). A method of partner selection for knowledge collaboration teams using weighted social network analysis, Journal of Intelligent Systems, Vol. 27, No. 4, 577-591, doi: 10.1515/iisys-2016$\underline{0140}$.

[17] Xiao, P., He L. (2014). Applications of logistical simulation to one-ladle-mode iron-making/steel-making interface, Steelmaking. Vol. 30, No. 4, 70-74.

[18] Xia, W., Song, W., Xia, Q. (2015). The application of system simulation and logistics automation technology in the steel production logistics process, In: Proceedings of the 2015 International Conference on Advanced Material Engineering, Guangzhou, China, 622-627, doi: 10.1142/9789814696029 0072.

[19] Han, W.-G., Li, X.-P., Shi, Y.-X, Wang, W.-D., Zhang, C.-X. (2013). Online ladle quantity of "one-open-ladle-from-BFto-BOF" route by using queuing theory, Iron \& Steel, Vol. 48, No. 5, 21-24.

[20] Huang, B.-F., Shi, Z., Zhou, X.-L., ZHANG, G.-F. (2015). Control model of torpedo ladle based on hot metal supply and demand, Journal of Kunming University of Science and Technology (Science and Technology), Vol. 40, No. 6, 133-138.

[21] Du, T., Cai, J.J., Li, Y.J., Wang, J.J. (2008). Analysis of hot metal temperature drop and energy-saving mode on techno-interface of BF-BOF route, Iron \& Steel, Vol. 43, No.12, 83-86.

[22] Ren, Y.-J., Wang, J.-W., Zhang, X.-B., Zhao, H.-W. (2012). Prediction model of hot metal temperature for BF-BOF interface based on LM BP neural network, Iron \& Steel, Vol. 47, No. 9, 40-39.

[23] Liu, S.W., Yu, J.K., Yan, Z.G., Liu, T. (2010). Factors and control methods of the heat loss of torpedo-ladle, Journal of Materials and Metallurgy, Vol. 9, No. 3, 159-163.

[24] Chen, D.M., Liu, Y.H., He, S.F., Xu, S., Dai, F.Q., Lu, B. (2020). Fuel gas operation management practices for reheating furnace in iron and steel industry, Advances in Production Engineering \& Management, Vol. 15, No. 2, 179-191, doi: 10.14743/apem2020.2.357.

[25] Chen, D., Lu, B., Chen, G., Yu, W. (2017). Influence of the production fluctuation on the process energy intensity in iron and steel industry, Advances in Production Engineering \& Management, Vol. 12, No. 1, 75-87, doi: 10.14743/ apem2017.1.241.

[26] Xiao, Y., Li, C., Song, L., Yang, J., Su, J. (2021) A multidimensional information fusion-based matching decision method for manufacturing service resource, IEEE Access, Vol. 9, 39839-39851, doi: 10.1109/ACCESS.2021.3063 $\underline{277}$. 


\section{APEM}

Advances in Production Engineering \& Management Letnik 16 | Številka 3 | September 2021 | Strani 348-358 https://doi.org/10.14743/apem2021.3.405
ISSN 1854-6250

Spletna stran: apem-journal.org Izvirni znanstveni članek

\title{
Na simulaciji temelječa optimizacija povezave materialno- energijskega toka pri železarsko-jeklarskem postopku z uporabo tehnike enega lonca
}

\author{
Hu, Z.C. ${ }^{a, b}$, Zheng, Z. ${ }^{a,}{ }^{*}$, He, L.M. ${ }^{c}$, Fan, J.P. ${ }^{b}$, Li, F. ${ }^{b}$ \\ aCollege of Materials Science and Engineering, Chongqing University, Chongqing, P.R. China \\ ${ }^{b} \mathrm{CISDI}$ Engineering Co., Ltd., Chongqing, P.R. China \\ 'Chongqing College of Electronic Engineering, Chongqing, P.R. China
}

\section{POVZETEK}

Železarsko-jeklarski postopek pri proizvodnji jekla vključuje kroženje lonca za vročo kovino in disipacijo energije, pri čemer gre za medsebojno povezana procesa, ki sta v neodvisnem razmerju. Zato je sinergijsko delovanje materialnega in energijskega toka v postopku ključnega pomena za učinkovito železarsko-jeklarsko proizvodnjo. Manjkajo pa rešitve za uresničitev boljše sinergije. $\mathrm{V}$ prispevku smo predstavili povezan simulacijski model pretoka materiala in energije v železarsko-jeklarskem postopku, ki temelji na matematičnem opisu možnega delovanja, trenutnega delovanja, tehničnem modelu proizvodne opreme ter modelu zniževanja temperature lonca. Nadalje je bil povezan simulacijski model uporabljen na železarsko-jeklarskem postopku s tehniko enega lonca. Povezan simulacijski model je dokazal svojo učinkovitost z zagotavljanjem celovite podpore pri odločanju, s predlogom optimiziranih strategij upravljanja proizvodnje in z rešitvijo, ki znižuje povprečen padec temperature vroče kovine za $10^{\circ} \mathrm{C}$ in zmanjšanje stroškov na tono jekla za 1,02 CNY.

\section{PODATKI O ČLANKU}

Ključne besede:

Metalurgija;

Postopek izdelave železa;

Postopek izdelave jekla;

Železarsko-jeklarski vmesnik;

Povezan materialno-energijski tok; Simulacija diskretnih dogodkov;

Optimizacija;

Tehnika enega lonca

*Kontaktna oseba:

zhengzh@cqu.edu.cn

(Zheng, Z.)

Zgodovina članka:

Prejet 8. avgusta 2021

Popravljen 17. septembra 2021

Sprejet 19. septembra 2021

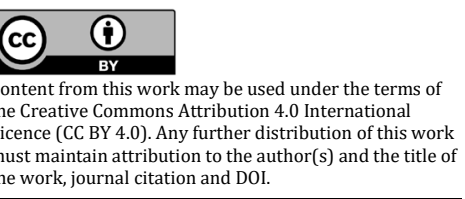

